\title{
El uso de corticoides inhalados en pacientes con EPOC se asoció a incremento del riesgo de neumonía grave
}

Inhaled corticosteroids increased the risk of serious pneumonia in patients with COPD

Suissa S. y col. Thorax 2013;68:1029-1036

\section{Objetivos}

Caracterizar el riesgo de neumonía severa asociado al uso de corticoides inhalatorios $(\mathrm{Cl})$ en pacientes con EPOC.

\section{Diseño, lugar y pacientes}

Estudio de casos y controles anidados sobre una cohorte de 163.514 pacientes mayores de 55 años con diagnóstico de EPOC, seleccionados de una base de datos de cobertura universal de salud de la provincia de Quebec, Canadá (RAMQ), entre 1990 a 2005. Se incluyeron pacientes a quienes se les dispensó en al menos dos fechas diferentes durante un año al menos tres prescripciones de cualquier forma de b2 agonistas, teofilina, ipratropio o tiotropio. Fueron excluidos los pacientes con asma o tratados con cualquier medicamento antiasmático. y seguidos hasta 2007. El seguimiento concluyo ante la ocurrencia de un episodio de neumonía severa (que requiriese hospitalización o produjese la muerte), fin de la cobertura social, o fecha de finalización del estudio (2007).

Evaluación de los factores de riesgo y medición de los resultados principales

Por cada caso de neumonía severa se identificaron diez controles apareados por edad. El factor de exposición se identificó cotejando la prescripción de un $\mathrm{Cl}$ (beclometasona, fluticasona, budesonide, triamcinolona y flunisolide), solo o en combinación con otra droga, entre la fecha de ingreso y el evento de interés. Las dosis de $\mathrm{Cl}$ se convirtieron a la equivalente de fluticasona en cuanto a potencia farmacológica y se clasificaron según la dosis usada en altas, moderadas y bajas $(1.000,500$ a 999 y $<500 \mathrm{mcg} /$ día, respectivamente)

\section{Resultados principales}

Se resumen en la tabla 1. La media de edad fue 72 años $(50 \%$ hombres) y la duración del seguimiento, 5,4 años. En ese período se identificaron 20.344 pacientes con neumonía severa, 19.667 se internaron y 677 fallecieron por neumonía. La incidencia de neumonía fue de 2,4 por 100 pacientes/año. Los usuarios de fluticasona presentaron el mayor riesgo de eventos (RR 2,01; IC95\% 1,93 a 2,10), y los usuarios de budesonide, el menor (RR 1,17; IC 95\% 1,09 a 1,26).

Tabla 1: riesgo relativo de neumonía severa asociada al uso de corticoides inhalados $(\mathrm{Cl})$ en pacientes con EPOC.

\begin{tabular}{l|c}
\multicolumn{1}{c|}{ Exposición a CI } & RR ajustado ${ }^{a}($ IC95\%) \\
\hline Sin uso previo $^{\beta}$ & 1,00 \\
\hline Uso vigente $^{*}$ & $1,69(1,63$ a 1,75$)$ \\
\hline Dosis baja & $1,24(1,13$ a 1,36$)$ \\
\hline Dosis moderada & $1,66(1,59$ a 1,74$)$ \\
\hline Dosis alta & $1,86(1,77$ a 1,94$)$ \\
\hline Uso pasado $\infty$ & $1,15(1,10$ a 1,20$)$ \\
\hline Suspensión 61 a 180 días & $1,19(1,13$ a 1,26$)$ \\
\hline Suspensión > 180 días & $1,08(0,99$ a 1,17$)$ \\
\hline
\end{tabular}

a Ajustado por edad, sexo, años de seguimiento, hospitalizaciones previas por EPOC o neumonía, medicación utilizada el año previo. $\beta$ Sin uso de un $\mathrm{Cl}$ dentro del año del evento índice. $¥$ Uso de un $\mathrm{Cl}$ dentro de los 60 días del evento índice. $\infty$ Uso de un $\mathrm{Cl}>60<360$ días del evento índice.

\section{Conclusiones}

El uso de $\mathrm{Cl}$ en pacientes con EPOC aumenta el riesgo de neumonía severa. El riesgo es particularmente elevado y se relaciona con la dosis de fluticasona, se sostiene con el uso cróni$\mathrm{co}$, declina luego de la suspensión de $\mathrm{Cl}$ y desaparece luego de seis meses.

\section{Comentario}

Se conoce que los corticoesteroides sistémicos se han asociados al incremento del riesgo de neumonía en pacientes con artritis reumatoidea en dosis tan bajas como $5 \mathrm{mg} /$ día de prednisona ${ }^{1,2}$. Por ello no se considera inesperado el reporte de efectos similares en dosis de $1000 \mathrm{mcg}$ de fluticasona equivalentes a $10 \mathrm{mg} /$ día de prednisona. Los estudios INSPIRE y TORCH con 2 y 3 años de seguimiento evaluaron altas dosis de fluticasona y hallaron un incremento en el riesgo de neumonía del $64 \%$ y $94 \%$ respectivamente ${ }^{3,4}$. Otros dos estudios evaluaron dosis de fluticasona de $500 \mathrm{mcg} / \mathrm{día}$ durante un año y reportaron un incremento del riesgo de neumonía del doble ${ }_{5,6}$. Un análisis de varios trabajos sobre 7.000 pacientes que recibieron budesonide no encontró incrementos significativos del riesgo de neumonía, mientras que en dos metanálisis se reporta un incremento menor comparado con fluticasona ${ }^{7,8}$ Dentro de las limitantes del trabajo a pesar de los ajustes por severidad de EPOC y criterios de selección, algunos pacientes tratados con budesonide podrían ser asmáticos o EPOC leves.

\section{Conclusiones del comentador}

El manejo de la EPOC implica objetivos inmediatos como el alivio sintomático y otros a largo plazo como el manejo de las exacerbaciones, comorbilidades y la disminución de la mortalidad. Se deberá tener en cuenta el uso de $\mathrm{Cl}$ y en especial la fluticasona a la hora de la elección terapéutica en el paciente con EPOC.

Sergio Daniel Zunino [ Sección Neumonología, Servicio de Clínica médica del Hospital Italiano de Buenos Aires. sergio.zunino@hospitalitaliano.org.ar ]

Zunino SD. El uso de corticoides inhalados en pacientes con EPOC se asoció a incremento del riesgo de neumonía grave. Evid Act Pract Ambul. 2014:17(3).Jul-Sep. 84. Comentado de: S Suissa y col. Inhaled corticosteroids in COPD and the risk of serious pneumonia. Thorax 2013;68:1029-1036. PMID: 24130228.

\section{Referencias}

1. Wolfe $\mathrm{F}$ y col. Treatment for rheumatoid arthritis and the risk of hospitalization for pneumonia: associations with prednisone, disease-modifying antirheumatics drugs, and antitumor necrosis factor therapy. Arthritis rheum 2006:54: 628-34. PMID: 16447241.

2. Grijalva CG y col. Initiation of rheumatoid arthritis treatment and de risk of serious infections. Rheumatology (Oxford) 2010; 49:82-90. PMID: 19906833.

3. Crim C y col. Pneumonia risk in COPD patients receiving inhaled corticosteroid alone or in combination. TORCH study results. Eur Respir J 2009; 4:641-7. PMID 19443528.

4. Calverley PM y col. Reported pneumonia in patients with COPD:findings from the INSPIRE study. Chest 2011:139:505-12. PMID: 20576732.

5. Fergusson GT y col. Effect of fluticasone propionate/salmeterol (250/50 microg) or salmeterol (50 microg) on COPD exacerbations. Respir Med 2008;102:1099-108. PMID: 18614347.

6. Anzueto A y col. Effect of fluticasone propionate/salmeterol (250/50 microg) on COPD exacerbations and impact on patient outcomes. COPD 2009; 6: 320-9. PMID: 19863361.

7. SIN DD y col. Budesonide and the risk of pneumonia: a meta-analysis of individual patient data. Lancet 2009;374:712-19. PMID: 19716963.

8. Halpin DM y col. Budesonide/formoterol vs salmeterol/fluticasona in COPD: a systematic review and adjusted indirect comparison of pneumonia in randomised controlled trials. Int J Clin Pract 2011;65:764-74. PMID: 21676119. 